\title{
Unconventional superconductivity from magnetism in transition metal dichalcogenides
}

\author{
M. A. Rahimi and A. G. Moghaddam* \\ Department of Physics, Institute for Advanced Studies in Basic Sciences (IASBS), Zanjan 45137-66731, Iran \\ C. Dykstra, M. Governale ${ }^{\dagger}$ and U. Zülicke \\ School of Chemical and Physical Sciences and MacDiarmid Institute for Advanced Materials and Nanotechnology, \\ Victoria University of Wellington, PO Box 600, Wellington 6140, New Zealand
}

(Dated: November 10, 2018)

\begin{abstract}
We investigate proximity-induced superconductivity in monolayers of transition metal dichalcogenides (TMDs) in the presence of an externally generated exchange field. A variety of superconducting order parameters is found to emerge from the interplay of magnetism and superconductivity, covering the entire spectrum of possibilities to be symmetric or antisymmetric with respect to the valley and spin degrees of freedom, as well as even or odd in frequency. More specifically, when a conventional $s$-wave superconductor with singlet Cooper pairs is tunnel-coupled to the TMD layer, both spin-singlet and triplet pairings between electrons from the same and opposite valleys arise due to the combined effects of intrinsic spin-orbit coupling and a magnetic-substrate-induced exchange field. As a key finding, we reveal the existence of an exotic even-frequency triplet pairing between equal-spin electrons from different valleys, which arises whenever the spin orientations in the two valleys are noncollinear. All types of superconducting order turn out to be highly tunable via straightforward manipulation of the external exchange field.
\end{abstract}

\section{INTRODUCTION}

Understanding possible mechanisms for the coexistence and interplay of superconductivity with magnetism has been one of the most long-standing and intensely studied questions in condensed-matter physics. Conventional $s$-wave BCS superconductivity is known to be fragile against magnetic perturbations ${ }^{1,2}$ due to its spin-singlet character. Instances where $s$-wave superconductivity coexists with magnetism typically require spatially inhomogeneity. Pertinent examples are the theoretically proposed Fulde-Ferell-Larkin-Ovchinikov (FFLO) superconducting state ${ }^{3-5}$ that oscillates in space, or hybrid structures of superconducting and magnetic materials. ${ }^{6}$ Alternatively, unconventional $p$-wave superconductivity $^{7}$ can accommodate magnetism because it enables Cooper-pairing of electrons with the same spin. Originally developed to understand superfluidity in ${ }^{3} \mathrm{He},{ }^{8-11}$ and later surmised to describe superconducting order in $\mathrm{Sr}_{2} \mathrm{RuO}_{4},{ }^{12}$ triplet pairing has recently attracted particular attention in the context of proximity effects in superconductor-ferromagnet heterostructures. ${ }^{6,13,14}$ Especially intriguing features include the emergence of oddfrequency triplet pairing due to the interplay of superconducting correlations with inhomogeneous non-collinear magnetizations, ${ }^{6,13-19}$ and the possibility for exotic Majorana bound states to be hosted in systems where the three ingredients of spin-orbit-coupling, Zeeman splitting, and superconducting correlations are present. ${ }^{20-24}$

On the other hand, the experimental realization of atomically thin crystalline materials has opened up an entirely new era of mesoscopic and nanoscale physics. ${ }^{25-27}$ This development started with the isolation of graphene $e^{28-32}$ and rapidly continued with subsequent discoveries of related structures including silicene ${ }^{33,34}$ and transition metal dichalcogenide monolayers (TMD-
MLs) ${ }^{35,36}$ such as monolayer $\mathrm{MoS}_{2}$. TMD-MLs have been the focus of great interest due to the intriguing effects associated with the coupling of spin and valley degrees of freedom. ${ }^{37}$ Their very large intrinsic spin-orbit coupling (SOC) suggest these materials can be used as a building blocks for spintronic applications. ${ }^{38-43}$ Furthermore, a significant valley polarization can be induced by circularly polarized light, ${ }^{44-47}$ leading to the observation of the valley Hall effect and growing possibilities for various valleytronics and optoelectronics applications. ${ }^{48-50}$ Most recently, both experimental and theoretical investigations have revealed that monolayer and few layer TMDs exhibit superconducting signatures under certain circumstances. In particular, ionic-gated $\mathrm{MoS}_{2}, \mathrm{MoSe}_{2}$, and $\mathrm{WS}_{2}$ undergo a superconducting phase transition at intermediate dopings. ${ }^{51-53}$ Recent observations also revealed an unusual superconducting behavior in few-layer $\mathrm{NbSe}_{2}$ and $\mathrm{MoS}_{2}$ that is robust against very large inplane magnetic fields. ${ }^{54-56}$ This so-called Ising superconductivity originates from the intrinsic SOC and the twodimensional nature of these materials. ${ }^{57,58}$

Previous theoretical studies have focused on the proximity effect, topological intrinsic superconductivity, and the Josephson effect in TMD-ML. ${ }^{58-63}$ In the present work, we provide a complete understanding of the interplay of superconducting proximity effects with magnetism in TMD. Our model system consists of a TMDML that is coupled vertically to a superconducting material and also subject to a finite spin exchange induced by a ferromagnetic substrate or external magnetic field. The interplay of SOC, the two-valley band structure, and the externally induced spin splitting results in exotic proximity effects that are different from common superconductor-ferromagnet heterostructures. In particular, we show that spin-triplet pairings that are odd with respect to either frequency or the valley degree of freedom 

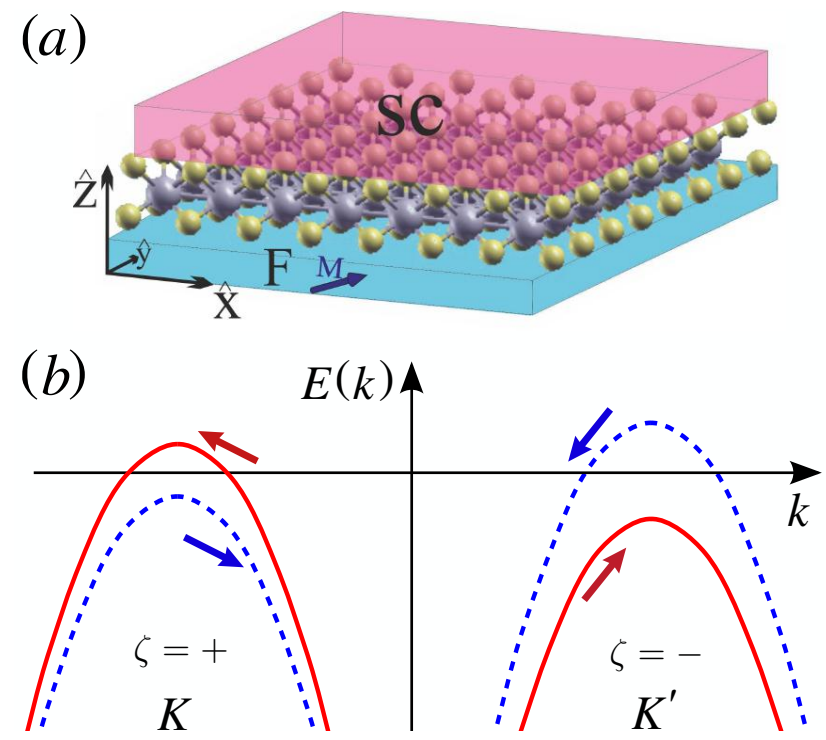

(c)
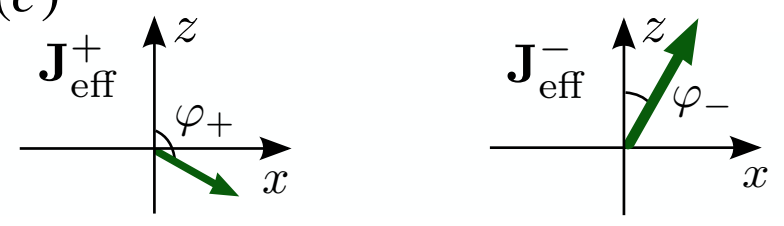

FIG. 1. Structure and properties of our system of interest. (a) Schematics of the hybrid superconductor-TMD-MLmagnetic-insulator system. (b) Valence-band structure of the TMD-ML in the presence of an externally induced exchange field $\mathbf{J}$. The spin states of each subband are indicated for an exchange field of the form $\mathbf{J}=\left(J_{x}, 0, J_{z}\right)$. (c) The effective exchange fields $J_{\text {eff }}^{\zeta}$ in the two valleys arising from the combination of $\mathbf{J}$ and SOC-induced spin splitting, which point at angles $\varphi_{ \pm}=\arctan \left[J_{x} /\left(J_{z} \mp \lambda\right)\right]$ with respect to the $z$ axis.

are induced without any inhomogeneous and noncollinear magnetizations. We also identify the existence of induced intra-valley pairings, which are particular instances of the generic pair-density-wave order associated with Cooper pairing at finite momentum ${ }^{5}$ that has been discussed previously, e.g., for high- $T_{\mathrm{c}}$ superconductors, ${ }^{64,65}$ other $2 \mathrm{D}$ materials, ${ }^{66-68}$ and Weyl semimetals. ${ }^{69}$

\section{BASIC THEORY AND FORMALISM}

We consider a TMD-ML on a ferromagnetic substrate that is tunnel-coupled to a conventional superconductor as sketched schematically in Fig. 1(a). The effect of the ferromagnetic substrate is to induce an exchange field $\mathbf{J}$ inside the TMD parallel to the substrate magnetization M. We focus on the case of a hole-doped TMD-ML and therefore only consider this material's two spin-split valence bands at each valley. The relevant band dispersions for this case are shown in Fig. 1(b). The low-energy Hamiltonian of this system in the vicinity of the $\mathbf{K}$ and
$\mathbf{K}^{\prime}$ points has the form

$$
\begin{aligned}
& \mathcal{H}_{\mathrm{TMD}}=\sum_{\mathbf{k}, \zeta} \hat{\boldsymbol{\phi}}_{\mathbf{k}, \zeta}^{\dagger}\left[\hat{h}_{\zeta}(\mathbf{k}) \otimes \hat{\tau}_{z}\right] \hat{\boldsymbol{\phi}}_{\mathbf{k}, \zeta} \\
& \hat{h}_{\zeta}(\mathbf{k})=E_{\mathbf{k}} \hat{\sigma}_{0}-\mathbf{J} \cdot \hat{\boldsymbol{\sigma}}+\zeta \lambda \hat{\sigma}_{z} \\
& E_{\mathbf{k}}=-\frac{\hbar^{2}|\mathbf{k}|^{2}}{2 m_{\mathrm{v}}}+E_{\mathrm{v}}
\end{aligned}
$$

The multi-component particle operator $\hat{\phi}_{\mathbf{k}, \zeta}^{\dagger}=$ $\left(c_{\mathbf{k} \zeta \uparrow}^{\dagger}, c_{\mathbf{k} \zeta \downarrow}^{\dagger}, c_{-\mathbf{k} \zeta \uparrow}, c_{-\mathbf{k} \zeta \downarrow}\right)$ is formed from the creation and annihilation operators $c_{\mathbf{k} \zeta s}^{\dagger}$ and $c_{\mathbf{k} \zeta s}$ for electron states with wave vector $\mathbf{k}$, spin $s=\uparrow, \downarrow$, and valley index $\zeta= \pm 1$ distinguishing the $\mathbf{K}$ and $\mathbf{K}^{\prime}$ points, respectively. The parameters $m_{\mathrm{v}}$ and $\lambda$ correspond to the valence-band effective mass and the strength of intrinsic spin-orbit coupling (SOC). The energy $E_{\mathrm{v}}$ measures the distance of the valence-band edge for vanishing $\mathbf{J}$ and $\lambda$ from the chemical potential of the superconductor. To be specific, we set $E_{\mathrm{v}}=0$ in the following, which implies that the chemical potential is in the middle between the spin-split valence bands. See Fig. 1(b). The Pauli matrices $\tau_{i}$ and $\sigma_{i}(i=x, y, z)$ operate on the Nambu and spin spaces, respectively. Corresponding identity matrices are denoted by $\tau_{0}$ and $\sigma_{0}$. The combination of the valley-antisymmetric SOC and the valley-symmetric exchange field leads to two different effective spin splittings in the $\mathbf{K}$ and $\mathbf{K}^{\prime}$ valleys,

$$
\mathbf{J}_{\mathrm{eff}}^{\zeta}=\mathbf{J}-\zeta \lambda \hat{\mathbf{z}}
$$

This is illustrated in Fig. 1(c).

The electronic degrees of freedom of a conventional $s$-wave superconductor are described by the mean-field BCS Hamiltonian

$$
\mathcal{H}_{\mathrm{SC}}=\sum_{\mathbf{q}} \hat{\boldsymbol{\psi}}_{\mathbf{q}}^{\dagger}\left(\xi_{\mathbf{q}} \hat{\sigma}_{0} \otimes \hat{\tau}_{z}-\Delta_{\mathrm{SC}} \hat{\sigma}_{y} \otimes \hat{\tau}_{y}\right) \hat{\boldsymbol{\psi}}_{\mathbf{q}}^{\dagger}
$$

where the particle operator $\hat{\boldsymbol{\psi}}_{\mathbf{q}}^{\dagger}=\left(d_{\mathbf{q} \uparrow}^{\dagger}, d_{\mathbf{q} \downarrow}^{\dagger}, d_{-\mathbf{q} \uparrow}, d_{-\mathbf{q} \downarrow}\right)$ is formed from creation and annihilation operators for electron states inside the superconductor. The gap and single-particle dispersion relation of the superconductor are denoted by $\Delta_{\mathrm{SC}}$ and $\xi_{\mathbf{q}}=\hbar^{2} q^{2} /\left(2 m_{\mathrm{S}}\right)-E_{\mathrm{FS}}$, with $E_{\mathrm{FS}}$ being the Fermi energy of electrons in the superconductor. The tunnel coupling between the TMD-ML and the superconductor is modelled by the tunnelling Hamiltonian

$$
\begin{aligned}
\mathcal{H}_{T} & =\sum_{\mathbf{q}, \mathbf{k}}\left(\hat{\phi}_{\mathbf{k},+}^{\dagger}, \hat{\phi}_{\mathbf{k},-}^{\dagger}\right) T_{\mathbf{k}, \mathbf{q}} \hat{\psi}_{\mathbf{q}}+\text { h.c. } \\
T_{\mathbf{k}, \mathbf{q}} & =\left(\begin{array}{l}
t_{\mathbf{k}, \mathbf{q},+} \hat{\sigma}_{0} \otimes \hat{\tau}_{z} \\
t_{\mathbf{k}, \mathbf{q},-} \hat{\sigma}_{0} \otimes \hat{\tau}_{z}
\end{array}\right),
\end{aligned}
$$

where $T_{\mathbf{k}, \mathbf{q}}$ is an $8 \times 4$ matrix that couples the electronic states from the two materials. We assume generally different tunnel couplings $t_{\mathbf{k}, \mathbf{q},+}$ and $t_{\mathbf{k}, \mathbf{q},-}$ for the two valleys $\mathbf{K}$ and $\mathbf{K}^{\prime}$, respectively. We will furthermore focus 
on the case of constant momentum-independent tunnel couplings $t_{\mathbf{k}, \mathbf{q}, \zeta} \equiv t_{\zeta}$.

In order to investigate the proximity-induced superconducting correlations in the TMD-ML arising from its tunnel coupling to the superconductor, we make use of the Matsubara Green's-function formalism. ${ }^{70}$ In the absence of tunneling, the Green's function of the superconductor is given by

$$
\begin{aligned}
& G_{\mathrm{SC}}^{0}\left(\mathbf{q}, i \omega_{n}\right)=\left(\begin{array}{cc}
\hat{g}\left(\mathbf{q}, i \omega_{n}\right) & \hat{f}\left(\mathbf{q}, i \omega_{n}\right) \\
\hat{f}^{\dagger}\left(\mathbf{q}, i \omega_{n}\right) & -\hat{g}\left(-\mathbf{q},-i \omega_{n}\right)
\end{array}\right) \\
& \hat{g}\left(\mathbf{q}, i \omega_{n}\right)=\frac{\left(i \omega_{n}+\xi_{\mathbf{q}}\right) \hat{\sigma}_{0}}{\left(i \omega_{n}\right)^{2}-\varepsilon_{\mathbf{q}}^{2}}, \quad \hat{f}(\mathbf{q}, \omega)=\frac{i \Delta_{\mathrm{SC}} \hat{\sigma}_{y}}{\left(i \omega_{n}\right)^{2}-\varepsilon_{\mathbf{q}}^{2}},
\end{aligned}
$$

where $\varepsilon_{\mathbf{q}}=\sqrt{\xi_{\mathbf{q}}^{2}+\Delta_{\text {SC }}^{2}}$ is the quasiparticle excitation spectrum. The bare Green's function $G_{\mathrm{TMD}}^{0}$ of the isolated TMD consist of four diagonal blocks, each having a certain valley index and corresponding to either electrons or holes,

$$
G_{\mathrm{TMD}}^{0}=\left(\begin{array}{cccc}
\hat{g}_{e}^{(+)} & 0 & 0 & 0 \\
0 & \hat{g}_{h}^{(+)} & 0 & 0 \\
0 & 0 & \hat{g}_{e}^{(-)} & 0 \\
0 & 0 & 0 & \hat{g}_{h}^{(-)}
\end{array}\right) .
$$

The blocks on the diagonal are $2 \times 2$ matrices in spin space, given by the expressions $\hat{g}_{e, h}^{(\zeta)}\left(\mathbf{k}, i \omega_{n}\right)=\left[i \omega_{n} \pm\right.$ $\left.h_{\zeta}(\mathbf{k})\right]^{-\mathbf{1}}$.

The full Green's function $\mathcal{G}_{\text {TMD }}$ of the TMD-ML in the hybrid system can be obtained by the Dyson equation

$$
\begin{aligned}
\mathcal{G}_{\mathrm{TMD}} & =G_{\mathrm{TMD}}^{0}+G_{\mathrm{TMD}}^{0} \Sigma \mathcal{G}_{\mathrm{TMD}} \\
\Sigma\left(\mathbf{k}, i \omega_{n}\right) & =\sum_{\mathbf{q}} T_{\mathbf{k}, \mathbf{q}} G_{\mathrm{SC}}^{0}\left(\mathbf{q}, i \omega_{n}\right) T_{\mathbf{k}, \mathbf{q}}^{\dagger}
\end{aligned}
$$

By means of Eq. (7a), the Green's function $\mathcal{G}_{\mathrm{TMD}}$ of the TMD-ML in the presence of tunnel coupling is found as

$$
\mathcal{G}_{\mathrm{TMD}}\left(\mathbf{k}, i \omega_{n}\right)=\left[\left(G_{\mathrm{TMD}}^{0}\left(\mathbf{k}, i \omega_{n}\right)\right)^{-1}-\Sigma\left(\mathbf{k}, i \omega_{n}\right)\right]^{-1} .
$$

However, for a weakly coupled TMD-ML/S hybrid system, we have $\mathcal{G}_{\mathrm{TMD}}\left(\mathbf{k}, i \omega_{n}\right) \approx G_{\mathrm{TMD}}^{0}\left(\mathbf{k}, i \omega_{n}\right)+$ $\delta \mathcal{G}_{\mathrm{TMD}}^{(2)}\left(\mathbf{k}, i \omega_{n}\right)$ to a good approximation, where

$$
\delta \mathcal{G}_{\mathrm{TMD}}^{(2)}\left(\mathbf{k}, i \omega_{n}\right)=G_{\mathrm{TMD}}^{0}\left(\mathbf{k}, i \omega_{n}\right) \Sigma\left(\mathbf{k}, i \omega_{n}\right) G_{\mathrm{TMD}}^{0}\left(\mathbf{k}, i \omega_{n}\right) .
$$

The superconducting correlations induced in the TMD are manifested by a non-vanishing anomalous part of $\delta \mathcal{G}_{\text {TMD }}^{(2)}$ given in Eq. (9). After some straightforward algebra, the intra-valley and inter-valley components of the anomalous Green's functions for the TMD-ML can be cast in the form

$$
\begin{aligned}
& \hat{\mathcal{F}}^{\zeta \zeta^{\prime}}\left(\mathbf{k}, i \omega_{n}\right)=t_{\zeta} t_{\zeta^{\prime}} \hat{g}_{e}^{(\zeta)}\left(\mathbf{k}, i \omega_{n}\right) \hat{f}\left(i \omega_{n}\right) \hat{g}_{h}^{\left(\zeta^{\prime}\right)}\left(\mathbf{k}, i \omega_{n}\right)(10 \mathrm{a}) \\
& \hat{f}\left(i \omega_{n}\right) \equiv \sum_{\mathbf{q}} \hat{f}\left(\mathbf{q}, i \omega_{n}\right)=\left(\begin{array}{cc}
0 & \bar{f}_{\mathrm{SC}}\left(i \omega_{n}\right) \\
-\bar{f}_{\mathrm{SC}}\left(i \omega_{n}\right) & 0
\end{array}\right)(10 \mathrm{~b})
\end{aligned}
$$

\section{RESULTS AND DISCUSSION}

The retarded anomalous Green's function can be obtained from Eq. (10a) by analytic continuation, i.e. $i \omega_{n} \rightarrow \omega+i 0^{+}$. In the following, for the sake of brevity we will omit to write the small imaginary part. Similarly to the usual conventions applied to superconducting order parameters, ${ }^{12}$ the retarded anomalous Green's function that represents the proximity-induced superconducting correlations can be decomposed into singlet and triplet components as

$$
\begin{aligned}
\hat{\mathcal{F}}^{\zeta \zeta^{\prime}}(\mathbf{k}, \omega) & =\left[d_{0}^{\zeta \zeta^{\prime}}(\mathbf{k}, \omega) \hat{\sigma}_{0}+\mathbf{d}^{\zeta \zeta^{\prime}}(\mathbf{k}, \omega) \cdot \hat{\boldsymbol{\sigma}}\right] i \hat{\sigma}_{y} \\
& \equiv\left(\begin{array}{cc}
-d_{x}+i d_{y} & d_{0}+d_{z} \\
-d_{0}+d_{z} & d_{x}+i d_{y}
\end{array}\right)
\end{aligned}
$$

The singlet and triplet components of the superconducting correlations are parametrized by the scalar $d_{0}$ and the vector $\mathbf{d}$, respectively. The components $d_{x}$ and $d_{y}$ describe equal-spin triplet pairing, while $d_{0}$ and $d_{z}$ correspond to opposite-spin pairing. Since the overall wave function of the Cooper pairs must be antisymmetric due to the fermionic nature of electrons, the superconducting order parameter (OP) can have different symmetries with respect to each single-particle degree of freedom. Equation (10a) is symmetric in $\mathbf{k}$, and the overall fermionic anti-symmetry is realised as shown in Table I where the OPs are classified according to their symmetries with respect to spin, valley, and frequency. In what follows, we will show how various exotic triplet components can be induced in a TMD-ML via proximity to an $s$-wave singlet superconductor and in the presence of an exchange splitting. In particular, intra-valley and inter-valley pairings with both opposite- and equal-spin components emerge as a result of the interplay of superconductivity with magnetic exchange and SOC.

We can express the various component of the anomalous Green's function as

\begin{tabular}{|l|c|c|c|}
\hline OP & spin & valley & $\omega$ \\
\hline$d_{0}^{(\mathrm{e})}$ & singlet & symmetric & even \\
\hline$d_{0}^{(\mathrm{o})}$ & singlet & antisymmetric & odd \\
\hline $\mathbf{d}^{(\mathrm{o})}$ & triplet & symmetric & odd \\
\hline $\mathbf{d}^{(\mathrm{e})}$ & triplet & antisymmetric & even \\
\hline
\end{tabular}

TABLE I. Classification of $s$-wave OPs according to symmetries in their spin and valley degrees of freedom, as well as their frequency dependence. 


$$
\begin{aligned}
d_{0}^{\zeta_{1} \zeta_{2}}(\mathbf{k}, \omega) & =t_{\zeta_{1}} t_{\zeta_{2}} \bar{f}_{\mathrm{SC}}(\omega)\left[\mathbf{J}_{\mathrm{eff}}^{\zeta_{1}} \cdot \mathbf{J}_{\mathrm{eff}}^{\zeta_{2}}+\omega^{2}-E_{\mathbf{k}}^{2}\right]\left[E^{\zeta_{1} \zeta_{2}}(\omega)+O^{\zeta_{1} \zeta_{2}}(\omega)\right] \\
\mathbf{d}^{\zeta_{1} \zeta_{2}}(\mathbf{k}, \omega) & =-t_{\zeta_{1}} t_{\zeta_{2}} \bar{f}_{\mathrm{SC}}(\omega)\left[\omega\left(\mathbf{J}_{\mathrm{eff}}^{\zeta_{1}}+\mathbf{J}_{\mathrm{eff}}^{\zeta_{2}}\right)+i \mathbf{J}_{\mathrm{eff}}^{\zeta_{1}} \times \mathbf{J}_{\mathrm{eff}}^{\zeta_{2}}+E_{\mathbf{k}}\left(\mathbf{J}_{\mathrm{eff}}^{\zeta_{1}}-\mathbf{J}_{\mathrm{eff}}^{\zeta_{2}}\right)\right]\left[E^{\zeta_{1} \zeta_{2}}(\omega)+O^{\zeta_{1} \zeta_{2}}(\omega)\right]
\end{aligned}
$$

where $\zeta_{1}$ and $\zeta_{2}$ are general valley indices, $E^{\zeta_{1} \zeta_{2}}(\omega)=$ $E^{\zeta_{2} \zeta_{1}}(\omega)$ is an even function of $\omega$, and $O^{\zeta_{1} \zeta_{2}}(\omega)=$ $-O \zeta_{2} \zeta_{1}(\omega)$ is an odd function of $\omega$. The functions $E^{\zeta_{1} \zeta_{2}}(\omega)$ and $O^{\zeta_{1} \zeta_{2}}(\omega)$ can be written as

$$
\begin{aligned}
& E^{\zeta_{1} \zeta_{2}}(\omega)=\frac{1}{2}\left(\frac{1}{\Gamma^{\zeta_{1} \zeta_{2}}(\omega)}+\frac{1}{\Gamma^{\zeta_{2} \zeta_{1}}(\omega)}\right), \\
& O^{\zeta_{1} \zeta_{2}}(\omega)=\frac{1}{2}\left(\frac{1}{\Gamma^{\zeta_{1} \zeta_{2}}(\omega)}-\frac{1}{\Gamma^{\zeta_{2} \zeta_{1}}(\omega)}\right),
\end{aligned}
$$

with

$\Gamma^{\zeta_{1} \zeta_{2}}(\omega)=\left[\left(\mathbf{J}_{\text {eff }}^{\zeta_{1}}\right)^{2}-\left(\omega-E_{\mathbf{k}}\right)^{2}\right]\left[\left(\mathbf{J}_{\text {eff }}^{\zeta_{2}}\right)^{2}-\left(\omega+E_{\mathbf{k}}\right)^{2}\right]$.

The form of Eqs. (12) enables straightforward identification of superconducting correlations that are even or odd in frequency. We then define order parameters for the various even-frequency superconducting correlations via their $\omega \rightarrow 0$ limit; $\hat{\mathcal{F}} \zeta \zeta^{\prime}(\mathbf{k}, \omega=0)$. Similarly, the order parameters of odd-frequency pairings are defined as the $\omega \rightarrow 0$ limit of their derivative w.r.t. frequency; $\left.\partial_{\omega} \hat{\mathcal{F}}^{\zeta \zeta^{\prime}}(\mathbf{k}, \omega)\right|_{\omega=0}$. In the following, we discuss the cases of proximity-induced intra-valley $\left(\zeta_{1}=\zeta_{2}\right)$ and intervalley $\left(\zeta_{1}=-\zeta_{2}\right)$ pair correlations, separately.

\section{A. Intra-valley pairing}

At first sight, intra-valley superconducting pairing may seem counter-intuitive because it implies Cooper pairs having finite momentum. However, the existence of such pair correlations is not forbidden by fundamental symmetries, ${ }^{5,64}$ and instances of pair-density-wave order within individual valleys have been pointed out recently for graphene, ${ }^{66,67}$ Weyl semimetals, ${ }^{69}$ and a generic multivalley model system. ${ }^{68}$ Setting $\zeta_{1}=\zeta_{2} \equiv \zeta$ in Eqs. (12a) and $(12 \mathrm{~b})$ yields the following results:

$$
\begin{aligned}
& d_{0}^{\zeta \zeta}(\mathbf{k}, \omega)=t_{\zeta}^{2} \bar{f}_{\mathrm{SC}}(\omega) \frac{\left(\mathbf{J}_{\mathrm{eff}}^{\zeta}\right)^{2}+\omega^{2}-E_{\mathbf{k}}^{2}}{\Gamma^{\zeta \zeta}(\omega)}, \\
& \mathbf{d}^{\zeta \zeta}(\mathbf{k}, \omega)=t_{\zeta}^{2} \bar{f}_{\mathrm{SC}}(\omega) \frac{-2 \omega \mathbf{J}_{\mathrm{eff}}^{\zeta}}{\Gamma^{\zeta \zeta(\omega)}},
\end{aligned}
$$

where we have used $E^{\zeta \zeta}(\omega)=1 / \Gamma^{\zeta \zeta}(\omega)$ and $O^{\zeta \zeta}(\omega)=0$. Clearly, the singlet and triplet components for intravalley pairing are even- and odd-frequency, respectively. This is consistent with the fact that the even symmetry of the components $d_{x, y, x}^{\zeta \zeta}$ with respect to the valley degree of freedom requires odd symmetry with respect to the frequency according to Table I. Note, however, that the intra-valley pairings from the $\mathbf{K}$ and $\mathbf{K}^{\prime}$ points are generally different because of the valley dependence of the effective spin splitting parametrized by $\mathbf{J}_{\text {eff }}^{\zeta}$, as illustrated in Fig. 1(c), and potentially also because of a valley-dependent tunnel coupling $t_{\zeta}$. The proportionality of the triplet pairing vector $\mathbf{d}^{\zeta \zeta}$ to the effective spin splitting $\mathbf{J}_{\text {eff }}^{\zeta}$ in each valley renders this exotic order parameter highly tunable. In particular, $d_{x, y}^{\zeta \zeta}$ will only be finite in the presence of finite external exchange-field components $J_{x, y}$. In contrast, SOC results in a finite $d_{z}^{\zeta \zeta}$ even in the absence of any exchange field.

To illustrate the parametric dependences of the intravalley order parameters, we consider the quantities,

$$
\begin{gathered}
\tilde{d}_{0}^{\zeta}=\frac{d_{0}^{\zeta \zeta}(\mathbf{k}, 0)}{t_{\zeta}^{2} \bar{f}_{\mathrm{SC}}(0)} \equiv\left[\left(\mathbf{J}_{\mathrm{eff}}^{\zeta}\right)^{2}-E_{\mathbf{k}}^{2}\right]^{-1}, \\
\tilde{\mathbf{d}}^{\zeta}=\frac{\left.\partial_{\omega} \mathbf{d}^{\zeta \zeta}(\mathbf{k}, \omega)\right|_{\omega=0}}{t_{\zeta}^{2} \bar{f}_{\mathrm{SC}}(0)} \equiv \frac{-2 \mathbf{J}_{\mathrm{eff}}^{\zeta}}{\left[\left(\mathbf{J}_{\mathrm{eff}}^{\zeta}\right)^{2}-E_{\mathbf{k}}^{2}\right]^{2}} .
\end{gathered}
$$

Both $\tilde{d}_{0}^{\zeta}$ and $\tilde{\mathbf{d}}^{\zeta}$ are representative because the frequency dependence of superconducting correlations near the chemical potential is weak. Figure 2 shows results obtained for the situation when $\mathbf{J}$ is in the $x z$ plane. For definiteness, we choose $\mathbf{k}$ such that $E_{\mathbf{k}}=-0.25 \lambda$, but the same qualitative behavior is exhibited for other values.
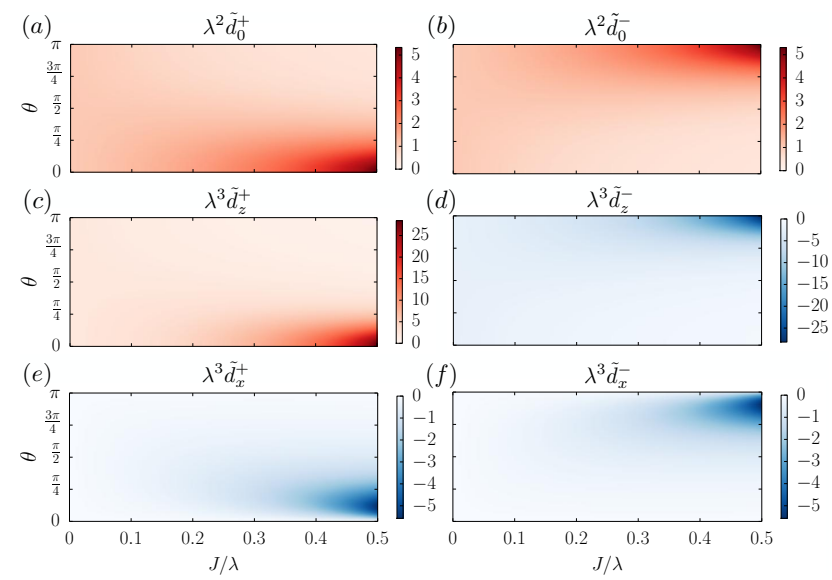

FIG. 2. Magnitude of intra-valley superconducting order parameters from Eqs. (15) induced in a monolayer TMD when the external exchange field is $\mathbf{J}=\left(J_{x}, 0, J_{z}\right)$. Here $J=\sqrt{J_{x}^{2}+J_{z}^{2}}, \theta=\arctan \left(J_{x} / J_{z}\right)$, and $E_{\mathbf{k}}=-0.25 \lambda$. 
Note that, to be able to plot dimensionless quantities, the effective order parameters that are even and odd in frequency have been scaled with $\lambda^{2}$ and $\lambda^{3}$, respectively.

The singlet pairings $\tilde{d}_{0}^{\zeta}$ are finite for any magnitude $J$ of the exchange field and direction parametrized by the angle $\theta=\arctan \left(J_{x} / J_{z}\right)$. However, the existence of triplet components $\tilde{d}_{z}^{\zeta}$ and $\tilde{d}_{x}^{\zeta}$, which are between opposite and equal spins, requires a finite exchange splitting $\mathbf{J}$ with out-of-plane and in-plane components, respectively. For large enough values of $J$, rapid increases in magnitude occur for all OPs whenever a divergence due to vanishing denominators in Eqs. (15) is approached. ${ }^{71}$ On the other hand, by changing the orientation of the external exchange field, the opposite-spin components at the two valleys reach maximum strengths for $\pm z$ alignments of $\mathbf{J}$, respectively. The strength of equal-spin pairings $d_{x}^{\zeta}$ can also be varied by $\theta$, and the maximum strengths for the different valleys $K$ and $K^{\prime}$ are attained for closeto-opposite out-of-plane alignments $\theta \gtrsim 0$ and $\theta \lesssim \pi$, respectively. Therefore, careful adjustment of the exchange field makes it possible to engineer situations where particular OP types dominate, rendering the hybrid system under consideration a versatile laboratory for studying unconventional superconductivity. Furthermore, the spin part of the triplet-Cooper-pair wave function $\left|\psi_{\mathrm{t}}^{\zeta}\right\rangle$ for each valley is fully determined by the vector $\mathbf{d}^{\zeta \zeta}$ as described, e.g., in Ref. 12. For the system under study the triplet wave functions can therefore be manipulated directly via the effective exchange fields as

$$
\begin{aligned}
\left|\psi_{\mathrm{t}}^{\zeta}\right\rangle \propto J_{\mathrm{eff}, x}^{\zeta}(-|\uparrow \uparrow\rangle+|\downarrow \downarrow\rangle) \\
\quad+i J_{\mathrm{eff}, y}^{\zeta}(|\uparrow \uparrow\rangle+|\downarrow \downarrow\rangle)+J_{\mathrm{eff}, z}^{\zeta}(|\uparrow \downarrow\rangle+|\downarrow \uparrow\rangle) .(16
\end{aligned}
$$

\section{B. Inter-valley pairing}

For the case $\zeta_{1}=-\zeta_{2} \equiv \zeta$, the proximity-induced superconducting pairings parametrized by Eqs. (12a) and (12b) exhibit a very rich behavior that can also be manipulated by the external exchange field $\mathbf{J}$. In particular, each order parameter has both even and odd-frequency components in general. The symmetries with respect to the spin and valley degrees of freedom, together with the even- or odd-frequency nature of the corresponding OPs, is consistent with the classifications given in Table I. Of particular interest is the emergence of the even-frequency equal-spin triplet component $\propto \mathbf{J}_{\text {eff }}^{\zeta} \times \mathbf{J}_{\text {eff }}^{-\zeta} \equiv 2 \zeta \lambda \mathbf{J} \times \hat{\mathbf{z}}$, which arises purely because of the noncollinearity of the effective spin-splitting fields in the two valleys and is therefore a direct consequence of the interplay between SOC and the external exchange field. Furthermore, an odd-frequency singlet OP emerges when the effective exchange fields for the two valleys are different. This is analogous to the situation encountered previously in double quantum-dot systems in contact with an $s$-wave superconductor. ${ }^{72,73}$

To illustrate again the parametric dependencies of the various OPs, we define the following quantities,

$$
\begin{aligned}
\tilde{d}_{0}^{(\mathrm{e})} & \equiv \frac{d_{0}^{\zeta,-\zeta}(\mathbf{k}, 0)}{t_{+} t_{-} \bar{f}_{\mathrm{SC}}(0)}=\frac{\mathbf{J}_{\text {eff }}^{+} \cdot \mathbf{J}_{\text {eff }}^{-}-E_{\mathbf{k}}^{2}}{\Gamma^{+-}(0)}, \\
\tilde{d}_{0}^{(o) \zeta} & \equiv \frac{\left.\partial_{\omega} d_{0}^{\zeta,-\zeta}(\mathbf{k}, \omega)\right|_{\omega=0}}{t_{+} t_{-} \bar{f}_{\mathrm{SC}}(0)}=\zeta\left(\mathbf{J}_{\text {eff }}^{+} \cdot \mathbf{J}_{\text {eff }}^{-}-E_{\mathbf{k}}^{2}\right) \frac{2 E_{\mathbf{k}}\left[\left(\mathbf{J}_{\text {eff }}^{+}\right)^{2}-\left(\mathbf{J}_{\text {eff }}^{-}\right)^{2}\right]}{\left[\Gamma^{+-}(0)\right]^{2}}, \\
\tilde{\mathbf{d}}^{(\mathrm{e}) \zeta} & \equiv \frac{\mathbf{d}^{\zeta,-\zeta}(\mathbf{k}, 0)}{t_{+} t_{-} \bar{f}_{\mathrm{SC}}(0)}=-\zeta \frac{i \mathbf{J}_{\text {eff }}^{+} \times \mathbf{J}_{\text {eff }}^{-}-2 \lambda E_{\mathbf{k}} \hat{\mathbf{z}}}{\Gamma^{+-}(0)}, \\
\tilde{\mathbf{d}}^{(o)} & \equiv \frac{\left.\partial_{\omega} \mathbf{d}^{\zeta,-\zeta}(\mathbf{k}, \omega)\right|_{\omega=0}}{t_{+} t_{-} \bar{f}_{\mathrm{SC}}(0)}=\frac{-2 \mathbf{J}}{\Gamma^{+-}(0)}-\left[i \mathbf{J}_{\text {eff }}^{+} \times \mathbf{J}_{\text {eff }}^{-}-2 \lambda E_{\mathbf{k}} \hat{\mathbf{z}}\right] \frac{2 E_{\mathbf{k}}\left[\left(\mathbf{J}_{\text {eff }}^{+}\right)^{2}-\left(\mathbf{J}_{\text {eff }}^{-}\right)^{2}\right]}{\left[\Gamma^{+-}(0)\right]^{2}},
\end{aligned}
$$

with $\Gamma^{+-}(0)$ defined by Eq. (13c). As is apparent from the above relations, the odd-frequency singlet $\mathrm{OP}$ and the even-frequency triplet component are antisymmetric with respect to the exchange of valleys and, consequently, have no corresponding intra-valley OP realization. In order to illustrate more clearly the structure of valleyodd inter-valley OPs, we write down the explicit form of the Cooper-pair wave function for the spin-triplet even- frequency component:

$$
\begin{array}{r}
\left|\psi_{\mathrm{t}}^{(e)}\right\rangle \propto \lambda(|+-\rangle-|-+\rangle) \otimes\left[i J_{x}(-|\uparrow \uparrow\rangle+|\downarrow \downarrow\rangle)\right. \\
\left.+J_{y}(|\uparrow \uparrow\rangle+|\downarrow \downarrow\rangle)+E_{k}(|\uparrow \downarrow\rangle+|\downarrow \uparrow\rangle)\right] .
\end{array}
$$

As expected from symmetry arguments given above, this wave function is a valley-singlet $\propto(|+-\rangle-|-+\rangle)$ and vanishes in the absence of SOC, as the latter is responsible for the valley-symmetry breaking.

Figures 3 and 4 show the dependences of opposite-spin 

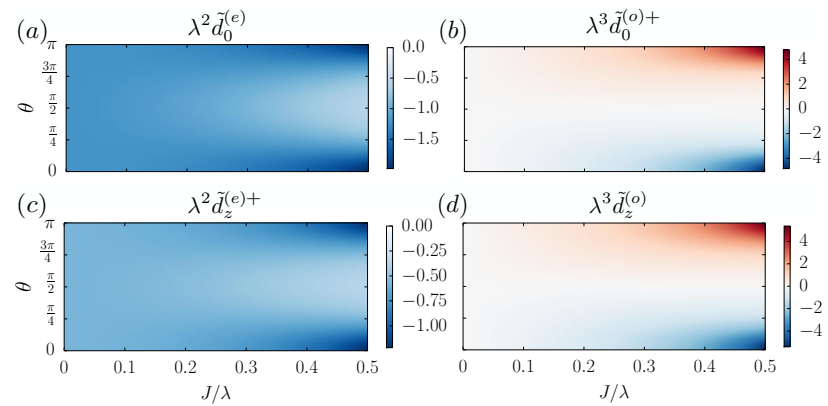

FIG. 3. Magnitude of inter-valley opposite-spin superconducting order parameters induced in a monolayer TMD when the external exchange field is $\mathbf{J}=\left(J_{x}, 0, J_{z}\right)$. Shown are dependences on $J=\sqrt{J_{x}^{2}+J_{z}^{2}}$ and $\theta=\arctan \left(J_{x} / J_{z}\right)$, keeping $E_{\mathrm{k}}=-0.25 \lambda$ fixed.

and equal-spin OPs, respectively, on the magnitude and direction of an external exchange field that is in the $x z$ plane. Again $E_{\mathbf{k}}=-0.25 \lambda$ was assumed when calculating these plots. While the even-frequency components $\tilde{d}_{0}^{(e)}$ and $\tilde{d}_{z}^{(e)}$ shown in Figs. 3(a),(c) are finite even for small $\mathbf{J}$, the corresponding odd-frequency OPs that can be seen in Figs. 3(b),(d) require the presence of an external exchange field. Moreover, all opposite-spin pairings have their largest magnitude for $\mathbf{J}$ perpendicular to the plane of the TMD-ML, i.e., when $\theta \sim 0$ and $\pi$.

Inspection of Fig. 4(a) reveals that the even-frequency equal-spin triplet OP represented by $\tilde{d}_{y}^{(\mathrm{e}) \zeta}$ has completely different behavior compared to opposite-spin terms shown in Fig. 3. In particular it becomes dominant in a broad range around $\theta \approx \pi / 2$ when $J / \lambda \gtrsim 0.2$, for the
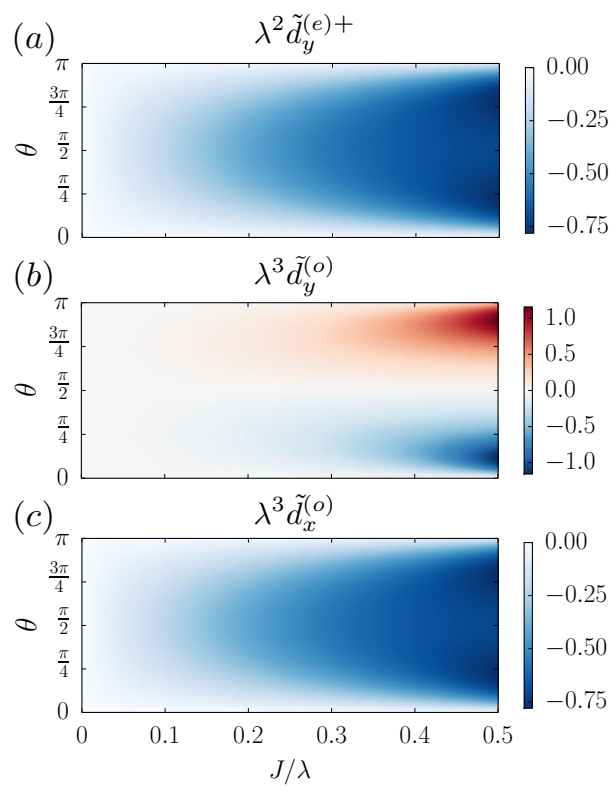

FIG. 4. Magnitude of inter-valley equal-spin superconducting order parameters for the same situation and conventions as in Fig. 3. chosen parameter regime. The odd-frequency equal-spin OP $\tilde{d}_{x}^{(\mathrm{o})}$ shown in Fig. 4(c) exhibits the same parametric dependences as $\tilde{d}_{y}^{(\mathrm{e}) \zeta}$, whereas $\tilde{d}_{y}^{(\mathrm{o})}$ [Fig. 4(b)] emerges only for large-enough $J / \lambda$ and for certain angles $\theta$ close to (but not equal to) 0 or $\pi$. Generally, the equal-spin OPs are only finite when $\mathbf{J}$ has a finite in-plane component. The plethora of OPs realized for inter-valley pairing is a direct consequence of the, in general, noncollinear effective exchange fields $\mathbf{J}_{\text {eff }}^{ \pm}$acting in the two valleys, which can again be tailored to generate specific superconducting pairing types.

\section{Discussion}

We have identified a great variety of superconducting OPs arising from the coupling of the TMD-ML to both magnetic and superconducting materials. Two basic ingredients are crucial for facilitating the unconventional types of pairing. First, the existence of two valleys enables symmetric and antisymmetric inter-valley pairing mechanisms so that even- and odd-frequency behavior becomes, in principle, possible for any type of superconducting OP. Secondly, the interplay of SOC and exchange splitting enables triplet pairings to emerge. Thus our system of interest differs markedly from previously considered superconductor-ferromagnet hybrid systems where a spatially nonuniform magnetization or a Rashba-type SOC gave rise to equal-spin triplet pairing. Our work also extends recent studies of intrinsic ${ }^{58,61}$ and proximityinduced $^{63}$ superconducting phases in a TMD-ML.

We find that the components of the exotic evenfrequency triplet OP $\tilde{\mathbf{d}}^{(\mathrm{e}) \zeta}$ can be comparable in magnitude to the conventional even-frequency spin-singlet OPs for intra- and inter-valley pairing. Separating $\tilde{\mathbf{d}}^{(\mathrm{e}) \zeta}=$ $\tilde{\mathbf{d}}_{\|}^{(\mathrm{e}) \zeta}+\tilde{\mathbf{d}}_{\perp}^{(\mathrm{e}) \zeta}$ in terms of the equal-spin and opposite-spin contributions

$$
\begin{aligned}
\tilde{\mathbf{d}}_{\|}^{(\mathrm{e}) \zeta} & =\frac{-2 i \zeta \lambda \mathbf{J} \times \hat{\mathbf{z}}}{\left[\left(\mathbf{J}_{\text {eff }}^{+}\right)^{2}-E_{\mathbf{k}}^{2}\right]\left[\left(\mathbf{J}_{\text {eff }}^{-}\right)^{2}-E_{\mathbf{k}}^{2}\right]}, \\
\tilde{\mathbf{d}}_{\perp}^{(\mathrm{e}) \zeta} & =\frac{2 \zeta \lambda E_{\mathbf{k}} \hat{\mathbf{z}}}{\left[\left(\mathbf{J}_{\text {eff }}^{+}\right)^{2}-E_{\mathbf{k}}^{2}\right]\left[\left(\mathbf{J}_{\text {eff }}^{-}\right)^{2}-E_{\mathbf{k}}^{2}\right]}
\end{aligned}
$$

the part $\tilde{\mathbf{d}}_{\perp}^{(\mathrm{e}) \zeta}$ can be identified with the OP previously associated with intrinsic Ising superconductivity ${ }^{58}$ The equal-spin part $\tilde{\mathbf{d}}_{\|}^{(\mathrm{e}) \zeta}$ has the same antisymmetric behavior with respect to the exchange of valleys, and its direct dependence on the exchange field $\mathbf{J}$ makes it highly tunable. Extending recent experimental studies ${ }^{54-56}$ of Ising superconductivity to situations where the TMDML is coupled to a magnetic insulator would facilitate exploration of this new OP. The prefactor $\lambda$ clearly indicates that finite SOC is an essential ingredient for both opposite-spin and equal-spin contributions to $\tilde{\mathbf{d}}^{(\mathrm{e}) \zeta}$.

It is worth noting that, generally, strong dependences on the valley index are exhibited by intra-valley OPs. 
This behavior results from the different effective exchange fields being present in each valley determining the pairing magnitudes. The valley-dependent proximityinduced superconductivity in the presence of a finite exchange field is a new manifestation of coupled spinvalley physics of TMDs. Thus complementing previous proposals for spintronics applications of TMD-MLs, our results indicate that hybrid systems made of magnetic TMD-MLs and conventional superconductors can be very promising systems for further investigations within the field of super-spintronics. ${ }^{74}$

Finally, we would like to comment on possible experimental observations of the effects found here. First of all, the setup we have proposed as a TMD-ML tunnelcoupled to a superconducting layer is realizable using current experimental techniques. Then, in order to recognize the various OPs having different symmetries with respect to spin, valley index and frequency, one should be able to identify the distinctive signatures of each component using, e.g., tunneling and Andreev spectroscopies, as these methods measure the local density of states and consequently can reveal the amplitude of the induced superconducting gap. ${ }^{75,76}$ For example, according to our results, one should observe an enhancement of the superconducting gap as the $z$ component of the external exchange splitting is increased, in contrast to the case of conventional superconductivity. On the other hand, the existence of odd-frequency OPs and their respective variations can also be observed using tunneling spectroscopy ${ }^{77,78}$ via the energy dependence of oddfrequency pairings and their tunability with the external exchange field. Furthermore, scanning Josephson spectroscopy ${ }^{79,80}$ can be employed to access the phase properties of the superconducting state. Lastly, spin-polarized scanning tunneling spectroscopy ${ }^{81}$ can reveal the presence of spin-triplet pairings through the suppression of Andreev reflection in the opposite-spin channel. ${ }^{82}$

\section{CONCLUSIONS}

We have investigated the proximity-induced superconductivity in monolayers of transition metal dichalcogenides that are tunnel-coupled to a conventional singlet $s$-wave superconductor and subject to a magnetizationinduced spin splitting. Various order parameters have been identified, exhausting all possible symmetries with respect to spin, valley, and frequency dependence. In particular, even-frequency inter-valley triplet pairing is facilitated by the intrinsic spin-orbit coupling in the transition metal dichalcogenide material. The opposite-spinpairing component of this exotic superconducting correlation is a realization of the previously discussed phenomenon of Ising superconductivity, while the equal-spin components are only finite when the exchange field has in-plane components.

We obtained analytical results that clearly illustrate the dependence of all possible superconducting order parameters on relevant parameters such as the magnitude and direction of the exchange field as well as the strength of the intrinsic spin-orbit coupling. Tailoring these parameters is shown to provide great selectivity to access dominant superconducting correlations in different regimes. We hope that this detailed insight into the behavior of an experimentally accessible system will facilitate further systematic exploration of exotic superconductivity in hybrid structures.

\section{ACKNOWLEDGMENTS}

A. G. M. acknowledges financial support from the Iran Science Elites Federation under Grant No. 11/66332.
* agorbanz@iasbs.ac.ir

† michele.governale@vuw.ac.nz

¥ uli.zuelicke@vuw.ac.nz

1 P. W. Anderson and H. Suhl, Phys. Rev. 116, 898 (1959).

2 L. N. Bulaevskii, A. I. Buzdin, M. L. Kulić, and S. V. Panjukov, Adv. Phys. 34, 175 (1985).

3 P. Fulde and R. A. Ferrell, Phys. Rev. 135, A550 (1964).

4 A. I. Larkin and Y. N. Ovchinnikov, Zh. Éksp. Teor. Fiz. 47, 1136 (1964), [Sov. Phys. JETP 20, 762 (1965)].

${ }^{5}$ R. Casalbuoni and G. Nardulli, Rev. Mod. Phys. 76, 263 (2004).

6 A. I. Buzdin, Rev. Mod. Phys. 77, 935 (2005).

7 V. P. Mineev and K. V. Samokhin, Introduction to Unconventional Superconductivity (Gordon and Breach, New York, 1999).

8 D. D. Osheroff, R. C. Richardson, and D. M. Lee, Phys. Rev. Lett. 28, 885 (1972).

9 V. L. Berezinskii, Pis'ma Zh. Éksp. Teor. Fiz. 20, 628 (1974), [JETP Lett. 20, 287 (1974)].
10 A. J. Leggett, Rev. Mod. Phys. 47, 331 (1975).

11 A. J. Leggett, Quantum Liquids: Bose Condensation and Cooper Pairing in Condensed-Matter Systems (Oxford University Press, Oxford, UK, 2006).

12 A. P. Mackenzie and Y. Maeno, Rev. Mod. Phys. 75, 657 (2003).

13 F. S. Bergeret, A. F. Volkov, and K. B. Efetov, Rev. Mod. Phys. 77, 1321 (2005).

14 R. S. Keizer, S. T. B. Goennenwein, T. M. Klapwijk, G. Miao, G. Xiao, and A. Gupta, Nature (London) 439, 825 (2006).

15 A. Kadigrobov, R. I. Shekhter, and M. Jonson, Europhys. Lett. 54, 394 (2001).

16 F. S. Bergeret, A. F. Volkov, and K. B. Efetov, Phys. Rev. Lett. 86, 4096 (2001).

17 A. F. Volkov, F. S. Bergeret, and K. B. Efetov, Phys. Rev. Lett. 90, 117006 (2003).

18 T. S. Khaire, M. A. Khasawneh, W. P. Pratt, and N. O. Birge, Phys. Rev. Lett. 104, 137002 (2010). 
19 J. W. A. Robinson, J. D. S. Witt, and M. G. Blamire, Science 329, 59 (2010).

${ }^{20}$ R. M. Lutchyn, J. D. Sau, and S. Das Sarma, Phys. Rev. Lett. 105, 077001 (2010).

21 Y. Oreg, G. Refael, and F. von Oppen, Phys. Rev. Lett. 105, 177002 (2010).

22 J. Alicea, Rep. Prog. Phys. 75, 076501 (2012).

${ }^{23}$ V. Mourik, K. Zuo, S. M. Frolov, S. R. Plissard, E. P. A. M. Bakkers, and L. P. Kouwenhoven, Science 336, 1003 (2012).

${ }^{24}$ C. W. J. Beenakker, Annu. Rev. Condens. Matter. Phys. 4, 113 (2013).

25 A. K. Geim and I. V. Grigorieva, Nature (London) 499, 419 (2013).

${ }^{26}$ M. Xu, T. Liang, M. Shi, and H. Chen, Chem. Rev. 113, 3766 (2013).

27 S. Z. Butler, S. M. Hollen, L. Cao, Y. Cui, J. A. Gupta, H. R. Gutiérrez, T. F. Heinz, S. S. Hong, J. Huang, A. F. Ismach, E. Johnston-Halperin, M. Kuno, V. V. Plashnitsa, R. D. Robinson, R. S. Ruoff, S. Salahuddin, J. Shan, L. Shi, M. G. Spencer, M. Terrones, W. Windl, and J. E. Goldberger, ACS Nano 7, 2898 (2013).

28 K. S. Novoselov, A. K. Geim, S. V. Morozov, D. Jiang, Y. Zhang, S. V. Dubonos, I. V. Grigorieva, and A. A. Firsov, Science 306, 666 (2004).

29 K. S. Novoselov, A. K. Geim, S. V. Morozov, D. Jiang, M. I. Katsnelson, I. V. Grigorieva, S. V. Dubonos, and A. A. Firsov, Nature (London) 438, 197 (2005).

30 Y. Zhang, Y.-W. Tan, H. L. Stormer, and P. Kim, Nature (London) 438, 201 (2005).

31 A. K. Geim and K. S. Novoselov, Nat. Mater. 6, 183 (2007).

32 A. H. Castro Neto, F. Guinea, N. M. R. Peres, K. S. Novoselov, and A. K. Geim, Rev. Mod. Phys. 81, 109 (2009).

33 S. Cahangirov, M. Topsakal, E. Aktürk, H. Şahin, and S. Ciraci, Phys. Rev. Lett. 102, 236804 (2009).

${ }^{34}$ P. Vogt, P. De Padova, C. Quaresima, J. Avila, E. Frantzeskakis, M. C. Asensio, A. Resta, B. Ealet, and G. Le Lay, Phys. Rev. Lett. 108, 155501 (2012).

${ }^{35}$ K. F. Mak, C. Lee, J. Hone, J. Shan, and T. F. Heinz, Phys. Rev. Lett. 105, 136805 (2010).

36 M. Chhowalla, H. S. Shin, G. Eda, L.-J. Li, K. P. Loh, and H. Zhang, Nat. Chem. 5, 263 (2013).

37 X. Xu, W. Yao, D. Xiao, and T. F. Heinz, Nat. Phys. 10, 343 (2014).

38 Z. Y. Zhu, Y. C. Cheng, and U. Schwingenschlögl, Phys. Rev. B 84, 153402 (2011).

39 H. Yuan, M. S. Bahramy, K. Morimoto, S. Wu, K. Nomura, B.-J. Yang, H. Shimotani, R. Suzuki, M. Toh, C. Kloc, X. Xu, R. Arita, N. Nagaosa, and Y. Iwasa, Nat. Phys. 9, $563(2013)$.

40 J. M. Riley, F. Mazzola, M. Dendzik, M. Michiardi, T. Takayama, L. Bawden, C. Granerød, M. Leandersson, T. Balasubramanian, M. Hoesch, T. K. Kim, H. Takagi, W. Meevasana, P. Hofmann, M. S. Bahramy, J. W. Wells, and P. D. C. King, Nat. Phys. 10, 835 (2014).

41 A. Kormányos, V. Zólyomi, N. D. Drummond, and G. Burkard, Phys. Rev. X 4, 011034 (2014).

42 R. Roldán, M. P. López-Sancho, F. Guinea, E. Cappelluti, J. A. Silva-Guillén, and P. Ordejón, 2D Materials 1, 034003 (2014).

43 J. Klinovaja and D. Loss, Phys. Rev. B 88, 075404 (2013).

${ }^{4}$ H. Zeng, J. Dai, W. Yao, D. Xiao, and X. Cui, Nat. Nanotech. 7, 490 (2012).
${ }^{45}$ K. F. Mak, K. He, J. Shan, and T. F. Heinz, Nat. Nanotech. 7, 494 (2012).

46 K. Behnia, Nat. Nanotech. 7, 488 (2012).

47 T. Cao, G. Wang, W. Han, H. Ye, C. Zhu, J. Shi, Q. Niu, P. Tan, E. Wang, B. Liu, and J. Feng, Nat. Comms. 3, 887 (2012).

${ }^{48}$ K. F. Mak, K. L. McGill, J. Park, and P. L. McEuen, Science 344, 1489 (2014).

49 D. Xiao, W. Yao, and Q. Niu, Phys. Rev. Lett. 99, 236809 (2007).

50 D. Xiao, G.-B. Liu, W. Feng, X. Xu, and W. Yao, Phys. Rev. Lett. 108, 196802 (2012).

51 J. T. Ye, Y. J. Zhang, R. Akashi, M. S. Bahramy, R. Arita, and Y. Iwasa, Science 338, 1193 (2012).

52 W. Shi, J. Ye, Y. Zhang, R. Suzuki, M. Yoshida, J. Miyazaki, N. Inoue, Y. Saito, and Y. Iwasa, Sci. Rep. 5, 12534 (2015).

53 S. Jo, D. Costanzo, H. Berger, and A. F. Morpurgo, Nano Lett. 15, 1197 (2015).

54 J. M. Lu, O. Zheliuk, I. Leermakers, N. F. Q. Yuan, U. Zeitler, K. T. Law, and J. T. Ye, Science 350, 1353 (2015).

55 X. Xi, Z. Wang, W. Zhao, J.-H. Park, K. T. Law, H. Berger, L. Forró, J. Shan, and K. F. Mak, Nat. Phys. 12, 139 (2016).

56 Y. Saito, Y. Nakamura, M. S. Bahramy, Y. Kohama, J. Ye, Y. Kasahara, Y. Nakagawa, M. Onga, M. Tokunaga, T. Nojima, Y. Yanase, and Y. Iwasa, Nat. Phys. 12, 144 (2016).

57 E. Navarro-Moratalla and P. Jarillo-Herrero, Nat. Phys. 12, $112(2016)$.

58 B. T. Zhou, N. F. Q. Yuan, H.-L. Jiang, and K. T. Law, Phys. Rev. B 93, 180501(R) (2016).

59 Y. Ge and A. Y. Liu, Phys. Rev. B 87, 241408(R) (2013).

60 R. Roldán, E. Cappelluti, and F. Guinea, Phys. Rev. B 88, 054515 (2013).

61 N. F. Q. Yuan, K. F. Mak, and K. T. Law, Phys. Rev. Lett. 113, 097001 (2014).

62 B. Z. Rameshti, A. G. Moghaddam, and M. Zareyan, Europhys. Lett. 108, 37002 (2014).

63 C. Triola, D. M. Badiane, A. V. Balatsky, and E. Rossi, Phys. Rev. Lett. 116, 257001 (2016).

${ }^{64}$ E. Fradkin, S. A. Kivelson, and J. M. Tranquada, Rev. Mod. Phys. 87, 457 (2015).

65 P. A. Lee, Phys. Rev. X 4, 031017 (2014).

66 B. Roy and I. F. Herbut, Phys. Rev. B 82, 035429 (2010).

67 J.-H. Zhou, T. Qin, and J.-R. Shi, Chin. Phys. Lett. 30 , 017401 (2013).

68 S. Tsuchiya, J. Goryo, E. Arahata, and M. Sigrist, Phys. Rev. B 94, 104508 (2016).

69 G. Y. Cho, J. H. Bardarson, Y.-M. Lu, and J. E. Moore, Phys. Rev. B 86, 214514 (2012).

70 H. Bruus and K. Flensberg, Many-Body Quantum Theory in Condensed Matter Physics: An Introduction (Oxford University Press, Oxford, UK, 2004).

${ }^{71}$ In real systems, such divergences are cut off by the finite quasiparticle lifetimes.

72 B. Sothmann, S. Weiss, M. Governale, and J. König, Phys. Rev. B 90, 220501 (2014).

73 P. Burset, B. Lu, H. Ebisu, Y. Asano, and Y. Tanaka, Phys. Rev. B 93, 201402 (2016).

74 J. Linder and J. W. A. Robinson, Nat. Phys. 11, 307 (2015). 
75 M. Tinkham, Introduction to Superconductivity, 2nd ed. (Dover Publications, Mineola, NY, 2004).

76 E. Wolf, Principles of Electron Tunneling Spectroscopy, 2nd ed. (Oxford University Press, Oxford, UK, 2012).

77 Y. Asano, Y. Tanaka, A. A. Golubov, and S. Kashiwaya, Phys. Rev. Lett. 99, 067005 (2007).

78 A. Di Bernardo, S. Diesch, Y. Gu, J. Linder, G. Divitini, C. Ducati, E. Scheer, M. Blamire, and J. W. A. Robinson,
Nat. Commun. 6, 8053 (2015).

79 J. Šmakov, I. Martin, and A. V. Balatsky, Phys. Rev. B 64, 212506 (2001).

80 M. T. Randeria, B. E. Feldman, I. K. Drozdov, and A. Yazdani, Phys. Rev. B 93, 161115 (2016).

81 R. Wiesendanger, Rev. Mod. Phys. 81, 1495 (2009).

${ }^{82}$ V. Kaladzhyan, P. Simon, and C. Bena, Phys. Rev. B 94, 134511 (2016). 